\title{
Novel Cocaine Vaccine Linked to a Disrupted Adenovirus Gene Transfer Vector Blocks Cocaine Psychostimulant and Reinforcing Effects
}

\author{
Sunmee Wee*,', Martin J Hicks², Bishnu P De ${ }^{2}$, Jonathan B Rosenberg ${ }^{2}$, Amira Y Moreno ${ }^{3}$, \\ Stephen M Kaminsky ${ }^{2}$, Kim D Janda ${ }^{3}$, Ronald G Crystal ${ }^{2}$ and George F Koob' \\ 'Committee on the Neurobiology of Addictive Disorders, The Scripps Research Institute, La Jolla, CA, USA; ${ }^{2}$ Department of Genetic Medicine, \\ Weill Cornell Medical College, New York, NY, USA; ${ }^{3}$ Department of Chemistry and Immunology, The Skaggs Institute for Chemical Biology, \\ La Jolla, CA, USA
}

\begin{abstract}
Immunotherapy is a promising treatment for drug addiction. However, insufficient immune responses to vaccines in most subjects pose a challenge. In this study, we tested the efficacy of a new cocaine vaccine (dAd5GNE) in antagonizing cocaine addiction-related behaviors in rats. This vaccine used a disrupted serotype 5 adenovirus (Ad) gene transfer vector coupled to a third-generation cocaine hapten, termed GNE (6-(2R,3S)-3-(benzoyloxy)-8-methyl-8-azabicyclo [3.2.1] octane-2-carboxamido-hexanoic acid). Three groups of rats were immunized with dAd5GNE. One group was injected with ${ }^{3} \mathrm{H}$-cocaine, and radioactivity in the blood and brain was determined. A second group was tested for cocaine-induced locomotor sensitization. A third group was examined for cocaine self-administration, extinction, and reinstatement of responding for cocaine. Antibody titers were determined at various time-points. In each experiment, we added a control group that was immunized with dAd5 without a hapten. The vaccination with dAd5GNE produced long-lasting high titers $\left(>10^{5}\right)$ of anti-cocaine antibodies in all of the rats. The vaccination inhibited cocaine-induced hyperlocomotor activity and sensitization. Vaccinated rats acquired cocaine self-administration, but they showed less motivation to self-administer cocaine under a progressive-ratio schedule than control rats. When cocaine was not available in a session, control rats exhibited 'extinction burst' responding, whereas vaccinated rats did not. Moreover, when primed with cocaine, vaccinated rats did not reinstate responding, suggesting a blockade of cocaine-seeking behavior. These data strongly suggest that our dAd5GNE vector-based vaccine may be effective in treating cocaine abuse and addiction.

Neuropsychopharmacology (2012) 37, 1083-1091; doi:I0.1038/npp.20 I 1.200; published online I4 September 20 I I
\end{abstract}

Keywords: cocaine; vaccine; rats; locomotor activity; self-administration; reinstatement

\section{INTRODUCTION}

One of the first reports of an immunotherapeutic approach to blunting the effects of drugs of abuse was the observation that immunization with a morphine vaccine decreased heroin self-administration in a rhesus monkey (Bonese et al, 1974). Since then, the feasibility of immunotherapy in treating drug abuse and addiction has been pursued across numerous drugs of abuse. An anti-nicotine vaccine was developed and tested in laboratory animals and proceeded to a clinical trial (Cerny, 2005; Keyler et al, 2008; Moreno et al, 2010). Vaccines against methamphetamine have been

*Correspondence: Dr S Wee, Committee on the Neurobiology of Addictive Disorders, SP30-2400, The Scripps Research Institute, 10550 North Torrey Pines Road, La Jolla, CA 92037, USA, Tel: + I 858784 7247, Fax: + I 858784 7405, E-mail: sunmee@scripps.edu Received 20 May 201।; revised 9 August 2011; accepted 10 August 2011 developed for the potential treatment of methamphetamine addiction (Duryee et al, 2009; Gentry et al, 2009; Xu et al, 2007). A heroin/morphine vaccine was also effective in decreasing heroin self-administration in rats (Anton and Leff, 2006; Anton et al, 2009).

Several vaccines against cocaine have been in development, with one tested in clinical trials (Carrera et al, 1995; Haney and Kosten, 2004; Kosten et al, 2002; Orson et al, 2009). For example, active immunization with a cocaine analog linked to keyhole limpet hemocyanin (KLH) significantly attenuated cocaine-induced psychomotor stimulation and sequestered cocaine in the periphery in rodents (Carrera et al, 1995, 2001). In addition, a KLHconjugated vaccine against cocaine prevented the reinstatement of responding for cocaine in rats, a rodent model of relapse to cocaine use (Carrera et al, 2000). Immunization with a cocaine-bovine serum albumin (BSA) conjugate also produced specific antibodies against cocaine and increased 
the distribution of cocaine in plasma relative to the brain in mice (Fox et al, 1996). Immunization with succinylnorcocaine attached to cholera B toxin (IPC-1010 or TA-CD) decreased responding for cocaine and cocaine intake in rats, with serum antibody concentrations $>0.05-0.07 \mathrm{mg} / \mathrm{ml}$ (Kantak et al, 2000, 2001), and this vaccine was well tolerated and produced specific antibodies in a dosedependent manner in a Phase I clinical trial (Kosten et al, 2002). In a Phase II clinical trial, $38 \%$ of the participants treated with TA-CD evoked antibody titer levels that correlated with lower cocaine levels in the urine, as an indicator of reduced cocaine use (Martell et al, 2009). In addition, subjects with TA-CD-induced high antibody titers reported significantly reduced subjective ratings after smoking cocaine (Haney et al, 2010). Therefore, despite the high individual variability in the immune response to vaccines, active immunization with cocaine conjugates appears to hold promise in the treatment of cocaine addiction.

Passive immunization with polyclonal or monoclonal antibodies was also effective in sequestering drugs of abuse in the periphery (McClurkan et al, 1993; Owens and Mayersohn 1986; Valentine et al, 1994; Valentine and Owens, 1996). Monoclonal antibodies against cocaine decreased cocaine self-administration and its toxic effects in rats (Carrera et al, 2000; Kantak et al, 2000; Mets et al, 1998). However, passively administered antibodies have a rather short half-life compared with active immunization that generates immunological memory (Fox et al, 1996; Kantak et al, 2000).

We previously reported that a cocaine vaccine that used a disrupted serotype 5 adenovirus gene transfer vector coupled to a first-generation cocaine hapten, GNC, remarkably reduced the psychomotor-stimulating effects of cocaine in mice, and antibody titers remained high for 3 months (Hicks et al, 2011). An Ad is a potent adjuvant that activates the immune system by infecting antigen-presenting cells. Therefore, by linking improved cocaine-hapten conjugates to Ad capsid proteins, we hypothesized that we would elicit high-level, high-affinity, cocaine-specific antibodies sufficient to treat cocaine addiction. The first-generation vaccine functioned well in mice. However, in our ongoing program, a third-generation cocaine hapten appeared to have a more potent immunological profile. In this study, therefore, we tested the effect of the vaccine that was developed using a third-generation cocaine hapten, termed GNE $(6-(2 R, 3 S)$ 3-(benzoyloxy)-8-methyl-8-azabicyclo [3.2.1] octane-2carboxamido-hexanoic acid), coupled to a disrupted serotype 5 Ad vector to create dAd5GNE. In this study, we tested the effect of dAd5GNE on cocaine-induced psychomotor stimulation, cocaine self-administration, and the reinstatement of responding for cocaine in rats, a rodent model of relapse in humans. In addition, throughout the study, we measured antibody titers to determine the duration of the vaccine titers and correlated these titers with behavioral measurements in rats.

We report here that the new vaccine generated high antibody titers in rats, which persisted for at least 4 months. The vaccination effectively prevented cocaine-induced locomotor activity and sensitization, the motivation to selfadminister cocaine, and cocaine seeking in rats. These data indicate that a disrupted Ad vaccine attached to an improved cocaine hapten may be effective in the treatment of cocaine abuse and addiction.

\section{MATERIALS AND METHODS}

All animal procedures were conducted in accordance with the National Institutes of Health Guide for the Care and Use of Laboratory Animals and were approved by the Institutional Animal Care and Use Committees of The Scripps Research Institute or Weill Cornell Medical College.

\section{Titer, Biodistribution, and Cocaine-Induced Hyperlocomotion}

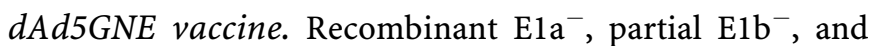
the $\mathrm{E}^{-}$serotype 5 Ad vector with $\beta$-galactosidase in the expression cassette ('Ad5') were expanded and purified (Rosenfeld et al, 1992). The Ad5 vector was disrupted in $0.5 \%$ sodium dodecyl sulfate $\left(56^{\circ} \mathrm{C}, 45 \mathrm{~s}\right)$. The cocaine hapten GNE $(0.3 \mathrm{mg})$ was activated overnight at $4^{\circ} \mathrm{C}$ after the addition of $7.2 \mu \mathrm{l}$ charging solution $(2.4 \mathrm{mg}$ of 1-ethyl-3(3-dimethylaminopropyl) carbodiimide hydrochloride and $2 \mathrm{mg}$ of $N$-hydroxysulfosuccinimide in $4 \mu \mathrm{l} \mathrm{H}_{2} \mathrm{O}$ and $40 \mu \mathrm{l}$ dimethylformamide). The conjugation of $200 \mu \mathrm{g}$ of disrupted Ad vector ('dAd5') with $67 \mu \mathrm{g}$ charged GNE (300:1 GNE to Ad capsomere molar ratio) was carried out by incubating overnight at $4{ }^{\circ} \mathrm{C}$ in $200 \mu$ l of phosphatebuffered saline (PBS, $\mathrm{pH}$ 7.4). The amount of Ad vector proteins was quantified using the bicinchoninic acid assay (Pierce Biotechnology, Rockford, IL).

Immunization with dAd5GNE (behavioral studies). Male Wistar (Hannover) rats ( $n=8$; Taconic, Germantown, NY) were vaccinated when they reached weights of 225-250 g. The rats were immunized at 0,3 , and 5 weeks (Figure 1) by intramuscular injection to the caudal thigh with $10 \mu \mathrm{g}$ of dAd5GNE in a $100 \mu \mathrm{l}$ volume, formulated in $20 \%$ Adjuplex (Advanced BioAdjuvants; LLC, Omaha, NE). Blood was

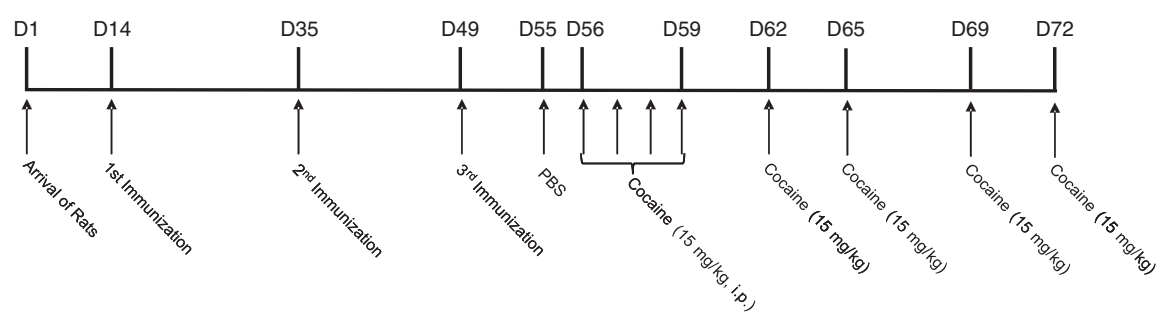

Figure I Timeline of cocaine ambulatory behavior studies. Day I (DI) through D72 for the vaccination and cocaine challenges that preceded the ambulatory behavior assays. 
collected from the tail vein, allowed to clot, and centrifuged at $10000 \mathrm{~g}$ for $20 \mathrm{~min}$. The isolated serum was stored at $-20^{\circ} \mathrm{C}$.

Assessment of anti-cocaine antibody levels. Wells of flatbottomed 96-well EIA/RIA plates (Corning, New York, NY) were coated with $100 \mu \mathrm{l}$ of $1 \mathrm{mg} / \mathrm{ml}$ cocaine hapten GNC conjugated to BSA (1:2 ratio) in carbonate buffer overnight at $4{ }^{\circ} \mathrm{C}$ using a conjugation method as described above. The plates were washed with $0.05 \%$ Tween 20 in PBS (PBSTween) and blocked with $5 \%$ dry milk in PBS for 30 min at $23^{\circ} \mathrm{C}$. Twofold serial dilutions of serum were added to each well and incubated for $90 \mathrm{~min}$ at $23^{\circ} \mathrm{C}$. The plates were washed four times with PBS-Tween. Total IgG was detected with $100 \mu \mathrm{l}$ of $1: 2000$ diluted horseradish peroxidaseconjugated goat anti-rat IgG (Santa Cruz Biotechnology) in $1 \%$ dry milk in PBS incubated for $90 \mathrm{~min}$ at $23^{\circ} \mathrm{C}$. Peroxidase substrate $(100 \mu \mathrm{l}$ per well; Bio-Rad, Hercules, CA) was added and incubated for $15 \mathrm{~min}$ at $23^{\circ} \mathrm{C}$. The peroxidase reaction was stopped with the addition of $2 \%$ oxalic acid $(100 \mu \mathrm{l}$ per well). Absorbance was measured at $415 \mathrm{~nm}$. Anti-cocaine antibody titers were calculated by interpolation of the $\log (\mathrm{OD})-\log$ (dilution), with a cutoff value equal to twice the absorbance of background.

Cocaine pharmacokinetics. dAd5GNE-vaccinated or -naive rats were anesthetized by intraperitoneal injection of ketamine $(80 \mathrm{mg} / \mathrm{kg})$ and xylazine $(8 \mathrm{mg} / \mathrm{kg}) 5 \mathrm{~min}$ before jugular administration of $25.0 \mu \mathrm{g}$ cocaine (National Institute on Drug Abuse drug supply program) with $3.0 \mu \mathrm{Ci}$ $\left[{ }^{3} \mathrm{H}\right]$ cocaine (Perkin-Elmer, Waltham, MA). At 2 min postadministration, the rats were killed, and the brain and trunk blood were collected separately. Brain tissue was homogenized in PBS and $500 \mu \mathrm{l}$ of brain homogenate, and $100 \mu \mathrm{l}$ of serum was added to separate $5 \mathrm{ml}$ liquid scintillation fluid (Ultima Gold; Perkin-Elmer), assayed in triplicate for tritium, and normalized with a standard quenching curve. The cocaine concentration from the blood compartment was normalized to serum volume and in the brain was normalized to brain wet weight.

Cocaine-induced locomotor sensitization. Rat locomotor activity was recorded using infrared beam-equipped openfield chambers $\left(40 \times 40 \mathrm{~cm}^{2}\right.$; Accusan Instruments, Columbus, $\mathrm{OH})$. Infrared beams were placed at $6 \mathrm{~cm}(X$ and $Y$ movements) and $20 \mathrm{~cm}$ ( $Z$ (vertical) movements) from the bottom of the cage. The rats were habituated to the room for $1 \mathrm{~h}$ before each test and were placed in the open-field chambers for $30 \mathrm{~min}$ to record baseline behavior. They were returned to their home cages for a maximum of $5 \mathrm{~min}$, injected with intraperitoneal PBS $(n=8)$ or cocaine $(15 \mathrm{mg} / \mathrm{kg})(n=7)$, and returned to the same chambers for $30 \mathrm{~min}$ to record post-treatment behavior. Locomotor activity was recorded as ambulatory distance traveled (centimeters) and the time spent (seconds) exhibiting vertical movement.

Statistical analysis. All data are expressed as mean \pm standard error. Comparisons between vaccinated and control groups were conducted using the paired $t$-test (Figure 2). Comparisons between the groups for each timepoint (challenge) were conducted using non-parametric one-way analysis of variance (ANOVA) and the KruskalWallis test. The Kruskal-Wallis test uses the mean score equal to the mean rank of each group (Figure 3), and the test statistic $(\mathrm{H})$ is approximately $\chi^{2}$ distributed. Comparisons between experimental groups for the analysis of multiple cocaine challenges were conducted using repeatedmeasures ANOVA (Figure 3). All statistical analyses were performed using Statview (version 5; SAS Institute, Cary, NC).

\section{Cocaine Self-Administration}

Apparatus. A standard operant chamber $\left(28 \times 26 \times 20 \mathrm{~cm}^{3}\right.$; Med Associates, St Albans, VT) was used for the experiments. The details of the operant chambers have been described previously (Wee et al, 2007). The start of a session was signaled by the extension of two response levers into the chamber. Responding on the right lever resulted in the delivery of $0.1 \mathrm{ml}$ of a drug injection over $4 \mathrm{~s}$. During an injection, a stimulus light above the active lever was illuminated and lasted throughout a $20 \mathrm{~s}$ time-out period that followed each injection when additional lever presses did not result in a drug infusion. Pressing the left lever was counted, but had no other programmed consequences.

dAd5GNE vaccination (self-administration studies). Male Wistar rats ( $n=12$; Charles River, Hollister, CA), weighing 225-250 $\mathrm{g}$ at the beginning of the study, were immunized at $0,3,5$, and 10 weeks (Figure 4) as described above. The
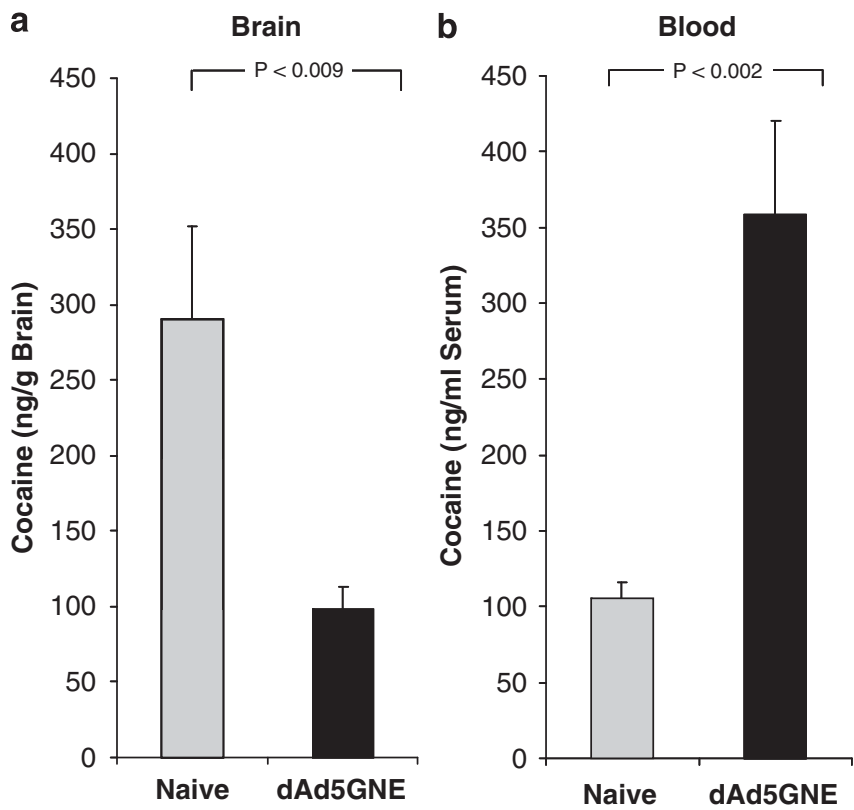

Figure 2 Brain and blood partition of cocaine in naive and dAd5GNE (vaccine that used a disrupted serotype 5 adenovirus (Ad) gene transfer vector coupled to a third-generation cocaine hapten, termed GNE (6-(2R,3S)-3-(benzoyloxy)-8-methyl-8-azabicyclo [3.2.I] octane-2-carboxamido-hexanoic acid))-vaccinated rats. (a) Levels of cocaine in the brain (ng/g brain). (b) Blood levels of cocaine ( $\mathrm{ng} / \mathrm{ml}$ serum) in naive and dAd5GNE-vaccinated rats challenged with cocaine. Cocaine assays were conducted at 2 min following the intravenous administration of cocaine (25.0 $\mu$ g containing $3.0 \mu \mathrm{Ci}^{3} \mathrm{H}$-cocaine) to rats 6 weeks after the fourth immunization with dAd5GNE $(n=5)$. Comparisons between groups were conducted by one-way paired two-sample t-test. 



Figure 3 Persistence of inhibition of cocaine-induced hyperlocomotor activity in dAd5GNE (vaccine that used a disrupted serotype 5 adenovirus (Ad) gene transfer vector coupled to a third-generation cocaine hapten, termed GNE (6-(2R,3S)-3-(benzoyloxy)-8-methyl-8-azabicyclo [3.2.I]
[ octane-2-carboxamido-hexanoic acid))-vaccinated rats. Comparison of naive and dAd5GNE-immunized rats assessed by cocaine-induced locomotor activity upon repeated exposures over an I8-day period with eight cocaine challenges. Vaccinated and naive + cocaine rats were sensitized to cocaine ( $15 \mathrm{mg} / \mathrm{kg}$, i.p.) during days $\mid-4$ and then challenged biweekly with cocaine ( $15 \mathrm{mg} / \mathrm{kg}$, i.p.) during days $7-18$ ( $n=8$ per group). (a) The total distance traveled inside an infrared beam-monitored openfield apparatus (AccuScan, $40 \times 40 \mathrm{~cm}^{2}$ ) for $30 \mathrm{~min}$ immediately following cocaine or phosphate-buffered saline (PBS) challenge was assessed for each trial. Rats from each experimental group were initially injected with PBS to establish baseline activity (data points at day 0). Comparisons between test groups used a non-parametric one-way analysis of variance (ANOVA) Kruskal-Wallis test: dAd5GNE + cocaine $(\mathbf{0})$ vs naive + cocaine ( $)$, $H=9.1, \quad p<0.01 ; \quad d A d 5 G N E+$ cocaine vs naive + PBS $(\Delta), \quad H=3.4$, $p>0.05$; naive + cocaine vs naive +PBS, $\mathrm{H}=\mid 1.3, p<0.001$. Repeatedmeasures ANOVA was performed on the biweekly challenges: dAd5GNE + cocaine vs naive + cocaine, $F=5.6, p<0.05 ; \mathrm{dAd} 5 \mathrm{GNE}+$ cocaine vs naive + PBS, $F=12.4, p<0.01$; naive + cocaine vs naive + PBS, $F=22.8$, $p<0.001$. The repeated-measures ANOVA within groups revealed no significant differences among the repetitions (dAdGNE, $F=0.8, p>0.5$; naive + cocaine, $F=0.2, p>0.5$; naive $+P B S, F=2.8, p>0.05$ ). (b) Total vertical activity time for $30 \mathrm{~min}$ immediately following $\mathrm{PBS}$ or cocaine ( $15 \mathrm{mg} / \mathrm{kg}$ ) challenge was plotted for each trial ( $n=8$ per group). The amount of time spent displaying vertical activity (ie, breaking $z$ axis beams) is plotted for each challenge event. Kruskal-Wallis test comparisons: dAd5GNE + cocaine (ם) vs naive + cocaine $(\mathbf{O}), \quad H=4.8, \quad p<0.05$; dAd5GNE + cocaine vs naive + PBS $(\Delta), H=0.5, p>0.1$; naive + cocaine vs naive + PBS, $H=5.8, p<0.05$. Repeated-measures ANOVA was performed on the biweekly challenges: dAd5GNE + cocaine vs naive + cocaine, $F=9.6, p<0.01$; dAd5GNE + cocaine vs naive $+P B S, F=0.2$, $p>0.5$; naive + cocaine vs naive $+P B S, F=13.3, p<0.01$. Repeatedmeasures ANOVA within groups for cocaine challenge revealed no significant differences $(F=0.1, p>0.5)$. control group of rats $(n=12)$ received the same treatment, but dAd5GNE was replaced with dAd5 (non-conjugated).

Effects of cocaine vaccine on the acquisition of cocaine self-administration. At 1 week after the last boost, the rats were implanted with indwelling catheters (Dow Corning, Midland, MI) into the right external jugular vein as described previously (Wee et al, 2007). One control rat was eliminated from the study because of surgical failure. At 1 week after the catheterization, they were allowed to selfadminister cocaine $(0.5 \mathrm{mg} / \mathrm{kg}$ per injection) for $1 \mathrm{~h}$ per day under a fixed-ratio 1 (FR1) schedule, in which one lever press resulted in a cocaine delivery. After 20 sessions of cocaine self-administration, a cocaine dose-response function was generated for each group. Specifically, in a test session, the baseline dose of cocaine was replaced with saline or $0.25,0.125$, or $0.0625 \mathrm{mg} / \mathrm{kg}$ per injection cocaine, with all of the other conditions identical. At least one baseline cocaine self-administration session $(0.5 \mathrm{mg} / \mathrm{kg}$ per injection) was inserted between two test sessions. The doses of cocaine were examined in an A-B-A design (0.5-0.250.125-0.0625-Sal-0.5). After the dose-response function of cocaine self-administration was determined, the rats were tested for cocaine self-administration under a progressive-ratio (PR) schedule. For the PR schedule, the response requirement began at one response per injection and increased according to the following equation: $\quad$ responses/injection $=\left(5 \times \mathrm{e}^{(\text {injection }}\right.$ number $\left.\left.\times 0.2\right)\right)-5$ (Richardson and Roberts, 1996). When a rat failed to achieve the response requirement within $1 \mathrm{~h}$, the session ended. At the end of the study, the rats were again allowed to self-administer cocaine for 2 days, after which methamphetamine $(0.05 \mathrm{mg} / \mathrm{kg}$ per injection) was substituted for cocaine for 2 days under the identical conditions. The rats were then tested for methamphetamine self-administration under a PR schedule for 2 days, and the PR performance on the second day was used for the data analysis. After methamphetamine self-administration, the rats were foodrestricted ( $15 \mathrm{~g}$ per day) and trained to self-administer food pellets ( $45 \mathrm{mg}$ Formula A/I; Research Diets, New Brunswick, $\mathrm{NJ}$ ) in a $15 \mathrm{~min}$ session under an FR1 schedule until the number of rewards reached was 100 rewards per $15 \mathrm{~min}$ for two consecutive sessions. The rats were then tested for food self-administration under a PR schedule. Methamphetamine and food self-administration under a PR schedule served as a control for the specific effect of vaccination on cocaine self-administration. Owing to catheter failures and health issues during the 3 months of the study, only 17 rats (control, $n=9$; vaccine, $n=8$ ) were tested for methamphetamine, and 16 rats ( $n=8$ per group) were tested in the food experiment.

Effects of cocaine vaccine on extinction and reinstatement of responding for cocaine. After the PR sessions, the rats were allowed to self-administer $0.5 \mathrm{mg} / \mathrm{kg}$ per injection of cocaine for an additional 4 days under baseline conditions (FR1, 1 h per day), after which extinction sessions were introduced. An extinction session lasted $2 \mathrm{~h}$. During an extinction session, a response on the active lever resulted in a saline injection without the cue light. After 10 extinction sessions, the rats were intraperitoneally injected with saline and immediately placed into an operant chamber to test for 


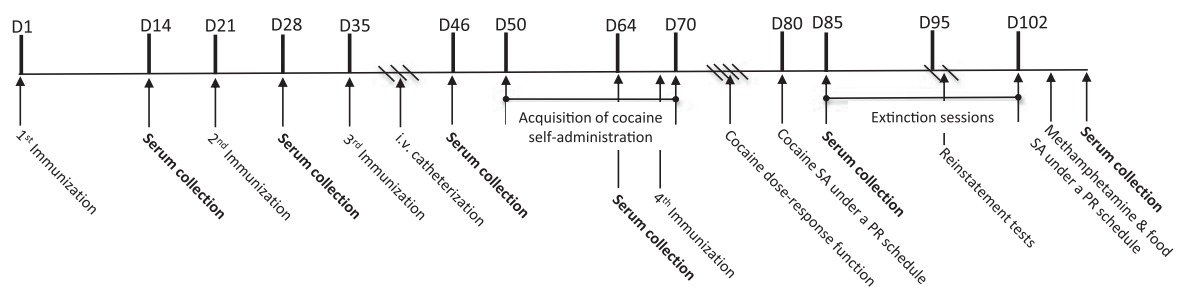

Figure 4 Timeline of cocaine self-administration. Day I (DI) through DI02 for the immunization, catheterization, and self-administration studies.

the reinstatement of responding as a control. The reinstatement session was identical to a cocaine self-administration session, except that a response on the active lever delivered saline, instead of cocaine, with a cue light on. Three extinction sessions followed the saline-induced reinstatement session, after which the rats were tested for cocaine-primed reinstatement of responding. The rats were intraperitoneally injected with 10 or $15 \mathrm{mg} / \mathrm{kg}$ of cocaine and immediately placed into the operant chambers to assess cocaine-primed reinstatement of responding. Three extinction sessions separated two cocaine-primed reinstatement sessions. Three rats (one control and two vaccinated) did not complete the reinstatement study because of compromised health. Therefore, they were excluded from the data analysis of reinstatement.

Statistical analysis. Cocaine self-administration is expressed as the number of cocaine injections ( \pm SEM). The effect of vaccination on the acquisition of cocaine self-administration for 20 days was examined using two-way repeated-measures ANOVA, followed by the Bonferroni post hoc test. In addition, nonlinear curve fitting of daily cocaine selfadministration for 20 days was used to compare differences in the initial level of cocaine self-administration, the plateau of cocaine self-administration, and the number of sessions $\left(\mathrm{ED}_{50}\right.$ session) required to reach half of the plateau of cocaine self-administration between groups. The effect of cocaine vaccination on the cocaine dose-response function under an FR schedule was examined using two-way repeated-measures ANOVA, followed by the Bonferroni post hoc test after the data were transformed to square root values. The data were transformed because of unequal variances in the number of cocaine injections across doses. In addition, the doseresponse functions of cocaine in both groups were fitted to linear regression lines after the $x$ axis was transformed in a log scale and were compared for a significant difference in the slopes and intercepts. The effect of vaccination on PR performance for cocaine, methamphetamine, and food was determined using Student's $t$-test. The effects of vaccination on the rate of extinction over the first eight sessions and on the reinstatement of responding were examined using twoway repeated-measures ANOVA, followed by the Bonferroni post hoc test. Prism 5 (GraphPad Software, La Jolla, CA) was used for all data analyses.

\section{RESULTS}

\section{Vaccine Administration, Titer, and Biodistribution}

The dAd5GNE vaccine evoked a strong immune response with anti-cocaine hapten ELISA reciprocal titer in a range

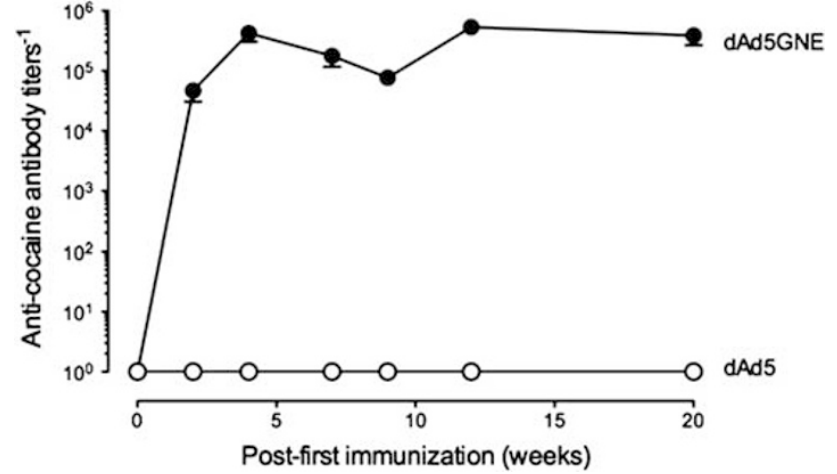

Figure 5 dAd5GNE (vaccine that used a disrupted serotype 5 adenovirus (Ad) gene transfer vector coupled to a third-generation cocaine hapten, termed GNE (6-(2R,3S)-3-(benzoyloxy)-8-methyl-8-azabicyclo [3.2.I] octane-2-carboxamido-hexanoic acid)) evoked anti-cocaine antibodies in rats. Total anti-cocaine immunoglobulin G (IgG) antibody titers over time. Male Wistar rats were vaccinated intramuscularly with $10 \mu \mathrm{g}$ dAd5GNE formulated in Aduplex or non-conjugated disrupted Ad5 (negative control). Rats $(n=12)$ were administered vaccine at $0,3,5$, and 10 weeks, and the antibody titers were assessed by enzyme-linked immunosorbent assay (ELISA) at 0, 2, 4, 7, 9, 12, 20, and 22 weeks. The data represent the animals used in the self-administration model.

from $2.8 \pm 0.3 \times 10^{5}$ to $9.4 \pm 2.4 \times 10^{5}$ (the locomotor activity experiment) and from $0.8 \pm 0.2 \times 10^{5}$ to $5.3 \pm 1.3 \times 10^{5}$ (the cocaine self-administration experiment; Figure 5) and a high affinity, $K_{\mathrm{d}}$, from 5 to $120 \mathrm{nM}$. When $\left[{ }^{3} \mathrm{H}\right]$ cocaine was administered intravenously via the jugular vein to the dAd5GNE rats, the levels in the brains of immunized rats were reduced by $66 \%$ compared with naive rats $(p<0.009)$. At the same time, the cocaine levels in the serum increased 3.5 -fold $(p<0.002$; Figure 2).

\section{Cocaine-Induced Locomotor Sensitization}

The anti-cocaine titers elicited by dAd5GNE vaccination prevented the induction of locomotor sensitization to each of the repeated administrations of cocaine $(15 \mathrm{mg} / \mathrm{kg})$, yielding serum levels in the rat comparable to typical human doses. Cocaine administered to naive rats induced ambulatory behavior more than twofold greater than dAd5GNE-vaccinated rats exposed to the same levels of cocaine $(H=9.1, p<0.003$; Figure $3 a)$. In addition, the repeated cocaine challenges to the naive rats resulted in a dramatic increase in the time spent exhibiting vertical activity, whereas similarly challenged dAd5GNE-vaccinated rats exhibited vertical activity similar to rats that received PBS instead of cocaine, which was significantly less than non-immunized rats $(H=0.5, p>0.45$; Figure $3 b)$. 
Evaluation of alternative behaviors revealed that cocaine did not increase stereotyped behavior as defined by the repeated breaking of the same photobeam at a frequency of greater than $1 \mathrm{~Hz}$ such that a $>1$-s interval between beam breaks initiates a new stereotyped episode (approx. 2-3\% of total activity).

\section{Cocaine Self-Administration}

Effects of cocaine vaccine on the acquisition of cocaine self-administration. When the rats were allowed to selfadminister $0.5 \mathrm{mg} / \mathrm{kg}$ per injection of cocaine, both groups of rats gradually increased the number of responses for cocaine $(2.3 \pm 0.4$ and $2.0 \pm 0.6$ to $14.8 \pm 1.7$ and $11.3 \pm 1.8$ injections per session in control and vaccine groups, respectively). There was no difference in the acquisition of cocaine self-administration across sessions between groups (Figure 6a).

Both groups of rats significantly increased their responses as the unit dose of cocaine for self-administration decreased (effect of dose, $\mathrm{F}_{3,3}=14, p<0.001$; Figure 6b). When analyzed using linear regression, both descending limbs of the dose-response function do not deviate from linearity (Runs test, $p>1.0$ ), and the slopes were significantly nonzero (control, $\mathrm{F}_{1,42}=7.4, p<0.01 ;$ dAd5GNE, $\mathrm{F}_{1,46}=4.9$, $p<0.05)$. However, more importantly, the elevations and intercepts of the linear regression lines were significantly different $\left(\mathrm{F}_{1,89}=5.4, p<0.05\right.$; Figure $\left.6 \mathrm{~b}\right)$, indicating a downward shift in the dose-response function of cocaine in the vaccinated rats, compared with that in the control rats. Under a PR schedule, the vaccinated rats exhibited significantly decreased responding for cocaine compared with control rats $(t=3.1, \mathrm{df}=21, p<0.01$; Figure $6 \mathrm{c})$. When substituted for cocaine, methamphetamine maintained $21 \pm 1.9$ and $16 \pm 2.0$ injections per $h$ in the control and vaccinated rats, respectively, under an FR schedule (Student's $t$-test, $p>0.05$ ). There was no difference between groups in PR performance for methamphetamine or food pellets (Table 1).

Effects of cocaine vaccine on extinction and reinstatement of responding for cocaine. When cocaine was replaced with saline in the sessions, the control rats showed a significantly higher level of responding on the previously cocaineassociated lever than the vaccinated rats in the first extinction session, but both groups significantly decreased their responding in eight sessions (Vaccination $\times$ Extinction Session interaction, $\mathrm{F}_{7,147}=2.4, p<0.05$; effect of Vaccination, $\mathrm{F}_{1,147}=6.6, p<0.05$; effect of Extinction Session, $\mathrm{F}_{7,147}=14, p<0.001$; Figure $7 \mathrm{a}$ ). When primed with cocaine immediately before a session, the control rats significantly reinstated responding on the previously cocaine-associated lever, whereas the vaccinated rats did not (effect of primed Cocaine, $\mathrm{F}_{2,36}=5.0, p=0.01$; Figure $7 \mathrm{~b}$ ).

\section{DISCUSSION}

This study demonstrated that a cocaine vaccine that used a disrupted Ad gene transfer vector elicited a robust and long-lasting humoral immune response in rats. We recently reported, using the same vector coupled to the first


Figure 6 Effect of dAd5GNE (vaccine that used a disrupted serotype 5 adenovirus (Ad) gene transfer vector coupled to a third-generation cocaine hapten, termed GNE (6-(2R,3S)-3-(benzoyloxy)-8-methyl-8-azabicyclo [3.2.I] octane-2-carboxamido-hexanoic acid)) on cocaine selfadministration. Rats were immunized with dAd5GNE ( $10 \mu \mathrm{g}$ per rat) and catheterized with intravenous catheters I week after the last boost. After recovery, they were allowed to self-administer $0.5 \mathrm{mg} / \mathrm{kg}$ per injection of cocaine in I h sessions under a fixed-ratio (FR) schedule. After 20 sessions, the cocaine dose-response function and responding for cocaine were determined under FR and progressive-ratio (PR) schedules, respectively. As a control, rats that were treated with dAd5 without a hapten underwent the same procedure as the vaccine group. At the end of the study, the rats were tested for food self-administration under a PR schedule. (a) Acquisition of cocaine self-administration under an FR schedule. The data are expressed as the number of cocaine injections on the left and cocaine intake $(\mathrm{mg} / \mathrm{kg}$ ) on the right. (b) Cocaine dose-response function. The data are expressed as the number of injections per hour at each dose of cocaine. The lines are linear regression lines. $* * * 0.01$, **** $p<0.00$ I, compared with responding for $0.5 \mathrm{mg} / \mathrm{kg}$ per injection of cocaine. (c) Cocaine self-administration under a PR schedule. The data are expressed as the number of injections per session on the left and ratio requirement per injection on the right. $* * x<0.0$ I, between groups. 
Table I Progressive-Ratio Performance for Methamphetamine and Food

\begin{tabular}{lcc}
\hline & $\begin{array}{c}\text { Number of rewards/session } \\
( \pm \text { SEM) }(\boldsymbol{n}=\mathbf{8 - 9})\end{array}$ \\
\cline { 2 - 3 } & $\begin{array}{c}\text { Vaccinated } \\
\text { group }\end{array}$ & $\begin{array}{c}\text { Non-vaccinated } \\
\text { group }\end{array}$ \\
\hline Methamphetamine $(0.05 \mathrm{mg} / \mathrm{kg}$ per inj.) & $8.4 \pm 1.5$ & $10.7 \pm 1.2$ \\
Food pellet & $10.8 \pm 0.9$ & $11.4 \pm 0.5$ \\
\hline
\end{tabular}
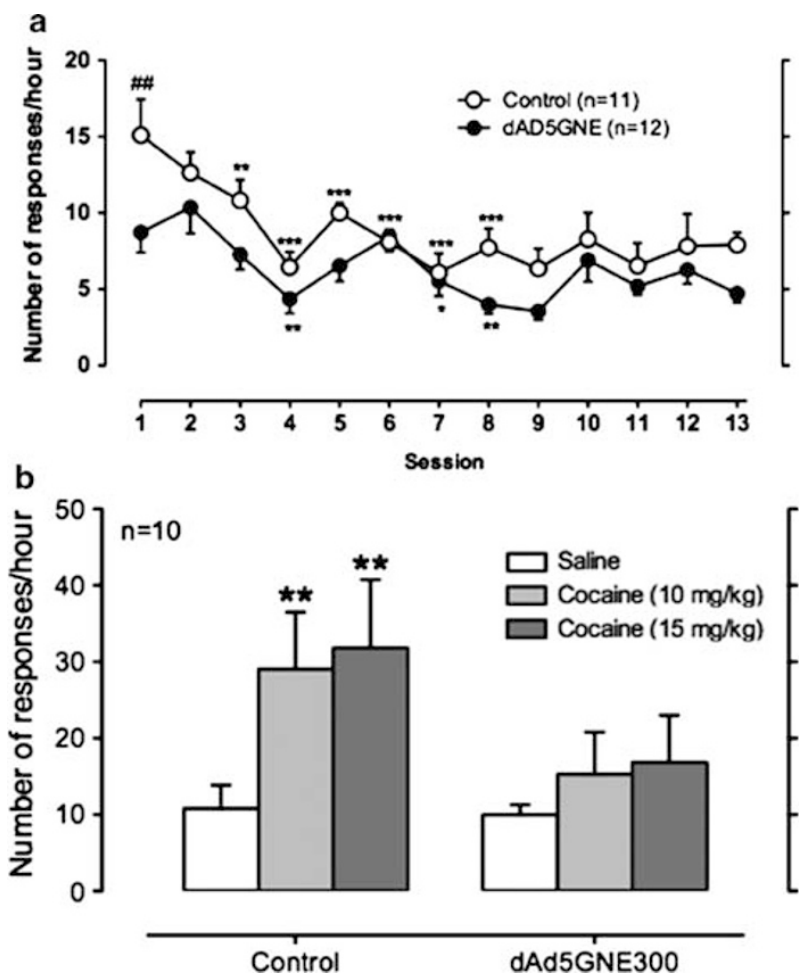

Figure 7 Extinction and reinstatement of responding for cocaine. During the extinction session, cocaine was replaced with saline, and responding on the previously cocaine-associated lever resulted in saline delivery without the cue light. For the reinstatement test, the rats were intraperitoneally injected with saline or cocaine ( 10 or $15 \mathrm{mg} / \mathrm{kg}$ ) and immediately placed in an operant chamber. During the reinstatement session, responding on the previously cocaine-associated lever resulted in saline delivery with the cue light. (a) Extinction. $* p<0.05$, $* * p<0.01$, **** $p<0.0$ I, compared with

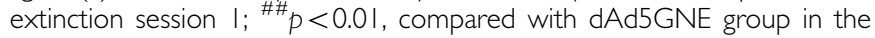
same session. (b) Reinstatement. $* * * 0.0$ I, compared with saline.

generation of a cocaine analog hapten (GNC), that the vaccine elicited a high level of antibody titers over 3 months in mice (Hicks et al, 2011). Therefore, disrupted adenovirus (dAd), a highly immunogenic cold virus, appears to be effective in producing strong immune responses against a non-immunogenic small-molecule drug in rodents. In sequestering drugs of abuse with antibodies, it is important to evoke both high titers and high-affinity antibodies to the drug to be able to counteract the bolus amount of a drug commonly used in addicts. In this study, the elicited antibodies increased the blood-to-brain ratio of cocaine distribution 7.5-fold, suggesting the functional specificity and efficacy of the antibodies to sequester free cocaine in the bloodstream sufficient to eliminate the subsequent cocaine-induced brain chemistry and subjective 'high'. Therefore, we predicted that immunization with dAd5GNE would inhibit the effects of cocaine on addiction-related behaviors in rats.

When acutely injected with cocaine, dAd5GNE-vaccinated rats displayed a vastly reduced response to cocaine-induced stimulant behaviors in all measurable categories. Although the cocaine injection in both the control and vaccinated groups elicited hyperlocomotion and rearing, the dAd5GNE-vaccinated groups exhibited a greatly reduced magnitude of these behaviors (Figure $3 a$ and $b$ ). In this study, the vaccinated rats showed a progressive decrease in the psychomotor-stimulating effect of cocaine during repeated injections of cocaine, whereas the non-vaccinated rats exhibited sensitization to the effect. Specifically, the hyperlocomotor activity that usually follows a high bolus dose of cocaine significantly decreased by $80 \%$ compared with naive control rats by the end of the experiment (ie, a 2.8-fold decrease; Figure 3a). Similarly, rats typically display hyperactivity of vertical (rearing) events in response to cocaine, which was diminished in vaccinated rats to the near-normal levels of PBS control rats (Figure 3b). The effect remained throughout eight challenges of $15 \mathrm{mg} / \mathrm{kg}$ cocaine over a 3-week period, demonstrating the persistence of vaccine efficacy and a lack of antibody exhaustion caused by multiple cocaine exposures. In fact, we found a high level of antibodies when measured 1 week after the last boost, which corresponded to the time course of the development of blockade of the psychostimulant effects of cocaine in the vaccinated rats.

Vaccination did not alter the initial acquisition of cocaine self-administration in rats. However, vaccinated rats showed a downward shift of the cocaine dose-response function compared with control rats, similar to a previous observation with succinylnorcocaine attached to cholera B toxin (Kantak et al, 2000). Most importantly, vaccinated rats exhibited decreased motivation to self-administer cocaine compared with control rats when the cost (ie, PR requirement) for a cocaine injection increased. This effect was specific to cocaine. Both the control and vaccinated groups showed no difference in their motivation to selfadminister methamphetamine and food. These PR results show a profile similar to lowering the dose of cocaine (Richardson and Roberts, 1996) and strongly suggest that vaccination decreases the reinforcing effects of cocaine. In humans, subjects with high cocaine antibody titers also reported significantly reduced 'good' drug effect and cocaine quality after smoking cocaine (Haney et al, 2010).

The finding that the vaccinated and control rats showed the same response to acute cocaine in the initial locomotor response to cocaine and no significant difference in the initial acquisition of cocaine self-administration deserves comment. One possible explanation for the locomotor activity may be the incomplete development of the control response to the drug combined with high spontaneous activity at these initial time-points. The locomotor response in the control animals is not particularly robust upon the initial injection of this modest dose of cocaine and only becomes substantial with repeated administration. We 
hypothesize that the initial hyperlocomotive response to cocaine may be less sensitive to blockade of cocaine by an antibody response as this behavior was less robust and thus less responsive to changes in cocaine dose. Similarly, acquisition of cocaine self-administration can occur over a wide range of doses, suggesting that this measure is also less sensitive to changes in dose. In contrast, sensitization of hyperlocomotion, PR performance, and drug-induced reinstatement of responding are particularly sensitive to a dose change in cocaine.

Consistent with the robust effects of the vaccine on motivation to seek cocaine in this study, we did not observe a 'compensatory-like' increase in cocaine self-administration in vaccinated rats as has been observed with low doses of dopamine antagonists and low antibody titers from other studies (Caine and Koob, 1994; Kantak et al, 2001). For example, previous results showed that passive immunization with a low concentration of monoclonal antibodies produced an upward shift of the cocaine dose-response function in rats, and a high concentration of monoclonal antibodies produced a downward shift as observed in this study (Kantak et al, 2001). Note again that all selfadministration testing in this study was performed after a complete immunization. Therefore, high stable antibody titers at the time of exposure to cocaine self-administration may have caused a downward shift, rather than a compensatory increase, in cocaine self-administration by vaccinated rats.

A major challenge in treating drug addiction is to prevent relapse to drug use after prolonged drug abstinence. The literature indicates that stress, drug-associated cues, and brief exposure to the drug itself can trigger drug craving and relapse to drug use in humans (Epstein et al, 2006; Yahyavi-Firouz-Abadi and See, 2009). Similarly, it is well established that animals that undergo extinction of drug self-administration reinstate responding when they are primed with a previously self-administered drug or other drugs of the same class (Stewart, 2000). This phenomenon is termed drug-primed reinstatement in animals. When saline was initially substituted for cocaine in this study, the control rats showed 'extinction burst' responding, which is commonly observed in actively learned responses (Harris et al, 2007), whereas the vaccinated rats did not. This observation is very similar to observations of 'extinction burst' responding in rats with high cocaine intake and no 'extinction bursts' in rats with low cocaine intake (Edwards et al, 2007). In addition, when primed with cocaine, control rats reinstated responding on the previously cocaineassociated lever, whereas vaccinated rats did not, consistent with a previous finding (Carrera et al, 2000). Consequently, the data suggest that the antibodies prevented primed cocaine from reaching the brain to initiate cocaine-seeking mechanisms in rats.

In summary, this study demonstrated that a disrupted Ad vector-based vaccine coupled to a third-generation cocaine hapten GNE was able to induce antibody titers at a sufficiently high level to antagonize the psychomotorstimulating and reinforcing effects of cocaine in rats. In addition, immunization with dAd5GNE inhibited cocaine seeking in rats when briefly exposed to cocaine after the extinction of cocaine self-administration. This study lasted approximately 4 months, supporting the long-lasting effect of dAd5GNE immunization in rats. Therefore, these data strongly suggest that a disrupted Ad gene therapy vectorbased vaccine may be effective in the treatment of cocaine abuse and addiction in humans.

\section{ACKNOWLEDGEMENTS}

We gratefully acknowledge Yanabel Grant for the excellent technical assistance. We thank Jeffrey Sobieraj, an undergraduate student intern from the University of California, San Diego, for assistance with cocaine self-administration and Michael Arends for editing the manuscript. We also thank the National Institute on Drug Abuse (NIDA) drug supply program for the cocaine used in this study. We thank Advanced BioAdjuvants, LLC of Omaha, NE for supplying Aduplex. This is publication number 21253 from The Scripps Research Institute. National Institute on Drug Abuse Grants DA004398 (GFK), DA025305 (RGC), and RC2 DA028847 (RGC) supported this study. SW is supported by K99 DA025785; MJH is supported, in part, by T32 HL094284; KDJ is supported, in part, by R01 DA008590; and JBR is partly supported by the National Foundation for Cancer Research, Bethesda, MD.

\section{DISCLOSURE}

All of the authors declare that they have no relationship with the organization supporting this research and no conflict of interest.

\section{REFERENCES}

Anton B, Leff P (2006). A novel bivalent morphine/heroin vaccine that prevents relapse to heroin addiction in rodents. Vaccine 24: 3232-3240.

Anton B, Salazar A, Flores A, Matus M, Marin R, Hernandez JA et al. (2009). Vaccines against morphine/heroin and its use as effective medication for preventing relapse to opiate addictive behaviors. Hum Vaccin 5: 214-229.

Bonese KF, Wainer BH, Fitch FW, Rothberg RM, Schuster CR (1974). Changes in heroin self-administration by a rhesus monkey after morphine immunization. Nature 252: 708-710.

Caine SB, Koob GF (1994). Effects of dopamine D-1 and D-2 antagonists on cocaine self-administration under different schedules of reinforcement in the rat. J Pharmacol Exp Ther 270: 209-218.

Carrera MR, Ashley JA, Parsons LH, Wirsching P, Koob GF, Janda KD (1995). Suppression of psychoactive effects of cocaine by active immunization. Nature 378: 727-730.

Carrera MR, Ashley JA, Wirsching P, Koob GF, Janda KD (2001). A second-generation vaccine protects against the psychoactive effects of cocaine. Proc Natl Acad Sci USA 98: 1988-1992.

Carrera MR, Ashley JA, Zhou B, Wirsching P, Koob GF, Janda KD (2000). Cocaine vaccines: antibody protection against relapse in a rat model. Proc Natl Acad Sci USA 97: 6202-6206.

Cerny T (2005). Anti-nicotine vaccination: where are we? Recent Results Cancer Res 166: 167-175.

Duryee MJ, Bevins RA, Reichel CM, Murray JE, Dong Y, Thiele GM et al. (2009). Immune responses to methamphetamine by active immunization with peptide-based, molecular adjuvant-containing vaccines. Vaccine 27: 2981-2988.

Edwards S, Whisler KN, Fuller DC, Orsulak PJ, Self DW (2007). Addiction-related alterations in D1 and D2 dopamine receptor 
behavioral responses following chronic cocaine self-administration. Neuropsychopharmacology 32: 354-366.

Epstein DH, Preston KL, Stewart J, Shaham Y (2006). Toward a model of drug relapse: an assessment of the validity of the reinstatement procedure. Psychopharmacology (Berlin) 189: 1-16.

Fox BS, Kantak KM, Edwards MA, Black KM, Bollinger BK, Botka AJ et al. (1996). Efficacy of a therapeutic cocaine vaccine in rodent models. Nat Med 2: 1129-1132.

Gentry WB, Ruedi-Bettschen D, Owens SM (2009). Development of active and passive human vaccines to treat methamphetamine addiction. Hum Vaccin 5: 206-213.

Haney M, Gunderson EW, Jiang H, Collins ED, Foltin RW (2010). Cocaine-specific antibodies blunt the subjective effects of smoked cocaine in humans. Biol Psychiatry 67: 59-65.

Haney M, Kosten TR (2004). Therapeutic vaccines for substance dependence. Expert Rev Vaccines 3: 11-18.

Harris AC, Pentel PR, Lesage MG (2007). Prevalence, magnitude, and correlates of an extinction burst in drug-seeking behavior in rats trained to self-administer nicotine during unlimited access (23 h/day) sessions. Psychopharmacology (Berlin) 194: 395-402. Hicks MJ, De BP, Rosenberg JB, Davidson JT, Moreno AY, Janda $\mathrm{KD}$ et al. (2011). Cocaine analog coupled to disrupted adenovirus: a vaccine strategy to evoke high-titer immunity against addictive drugs. Mol Ther 19: 612-619.

Kantak KM, Collins SL, Bond J, Fox BS et al. (2001). Time course of changes in cocaine self-administration behavior in rats during immunization with the cocaine vaccine IPC-1010. Psychopharmacology (Berlin) 153: 334-340.

Kantak KM, Collins SL, Lipman EG, Bond J, Giovanoni K, Fox BS (2000). Evaluation of anti-cocaine antibodies and a cocaine vaccine in a rat self-administration model. Psychopharmacology (Berlin) 148: 251-262.

Keyler DE, Roiko SA, Earley CA, Murtaugh MP, Pentel PR (2008). Enhanced immunogenicity of a bivalent nicotine vaccine. Int Immunopharmacol 8: 1589-1594.

Kosten TR, Rosen M, Bond J, Settles M, Roberts JS, Shields J et al. (2002). Human therapeutic cocaine vaccine: safety and immunogenicity. Vaccine 20: 1196-1204.

Martell BA, Orson FM, Poling J, Mitchell E, Rossen RD, Gardner T et al. (2009). Cocaine vaccine for the treatment of cocaine dependence in methadone-maintained patients: a randomized, double-blind, placebo-controlled efficacy trial. Arch Gen Psychiatry 66: 1116-1123.
McClurkan MB, Valentine JL, Arnold L, Owens SM (1993). Disposition of a monoclonal anti-phencyclidine Fab fragment of immunoglobulin G in rats. J Pharmacol Exp Ther 266: 1439-1445.

Mets B, Winger G, Cabrera C, Seo S, Jamdar S, Yang G et al. (1998). A catalytic antibody against cocaine prevents cocaine's reinforcing and toxic effects in rats. Proc Natl Acad Sci USA 95: 10176-10181.

Moreno AY, Azar MR, Warren NA, Dickerson TJ, Koob GF, Janda KD (2010). A critical evaluation of a nicotine vaccine within a self-administration behavioral model. Mol Pharmacol 7: 431-441.

Orson FM, Kinsey BM, Singh RA, Wu Y, Kosten TR (2009). Vaccines for cocaine abuse. Hum Vaccin 5: 194-199.

Owens SM, Mayersohn M (1986). Phencyclidine-specific Fab fragments alter phencyclidine disposition in dogs. Drug Metab Dispos 14: 52-58.

Richardson NR, Roberts DC (1996). Progressive ratio schedules in drug self-administration studies in rats: a method to evaluate reinforcing efficacy. J Neurosci Methods 66: 1-11.

Rosenfeld MA, Yoshimura K, Trapnell BC, Yoneyama K, Rosenthal ER, Dalemans W et al. (1992). In vivo transfer of the human cystic fibrosis transmembrane conductance regulator gene to the airway epithelium. Cell 68: 143-155.

Stewart J (2000). Pathways to relapse: the neurobiology of drugand stress-induced relapse to drug-taking. J Psychiatry Neurosci 25: $125-136$

Valentine JL, Arnold LW, Owens SM (1994). Anti-phencyclidine monoclonal Fab fragments markedly alter phencyclidine pharmacokinetics in rats. J Pharmacol Exp Ther 269: 1079-1085.

Valentine JL, Owens SM (1996). Antiphencyclidine monoclonal antibody therapy significantly changes phencyclidine concentrations in brain and other tissues in rats. J Pharmacol Exp Ther 278: 717-724.

Wee S, Specio SE, Koob GF (2007). Effects of dose and session duration on cocaine self-administration in rats. J Pharmacol Exp Ther 320: 1134-1143.

$\mathrm{Xu} \mathrm{Y}$, Hixon MS, Yamamoto N, McAllister LA, Wentworth AD, Wentworth P Jr et al. (2007). Antibody-catalyzed anaerobic destruction of methamphetamine. Proc Natl Acad Sci USA 104: 3681-3686.

Yahyavi-Firouz-Abadi N, See RE (2009). Anti-relapse medications: preclinical models for drug addiction treatment. Pharmacol Ther 124: $235-247$. 